\title{
Identification of a nuclear protein, LRRC42, involved in lung carcinogenesis
}

\author{
TAKASHI FUJTOMO $^{1}$, YATARO DAIGO ${ }^{1,2}$, KOICHI MATSUDA $^{1}$, KOJI UEDA $^{3}$ and YUSUKE NAKAMURA ${ }^{1,4}$ \\ ${ }^{1}$ Laboratory of Molecular Medicine, Human Genome Center, Institute of Medical Science, The University of Tokyo, \\ Tokyo 108-8639; ${ }^{2}$ Department of Medical Oncology and Cancer Center, Shiga University of Medical Science, \\ Otsu 520-2192; ${ }^{3}$ Laboratory for Biomarker Development, Center for Genomic Medicine, RIKEN, Yokohama 230-0045, \\ Japan; ${ }^{4}$ Department of Medicine, The University of Chicago, 5801 South Ellis Avenue, Chicago, IL 60637, USA
}

Received February 2, 2014; Accepted March 20, 2014

DOI: $10.3892 /$ ijo.2014.2418

\begin{abstract}
On the basis of the gene expression profiles of 120 lung cancer cases using a cDNA microarray containing 27,648 genes or expressed sequence tags (ESTs), we identified LRRC42 (Leucine-rich repeat containing 42) to be significantly upregulated in the majority of lung cancers. Northern blot analysis demonstrated that LRRC42 was expressed only in testis among normal tissues examined. Knockdown of LRRC42 expression by siRNA against LRRC42 significantly suppressed the growth of lung cancer cells. On the other hand, stable induction of LRRC42 expression significantly promoted cell growth. LRRC42, which was found to localize in the nucleus of mammalian cells, is likely to interact with and stabilize GATAD2B (GATA zinc finger domain-containing 2B) and MBD3 (Methyl-CpG-binding domain protein 3) proteins that could contribute to lung cancer cell proliferation partly through the regulation of $\mathrm{p} 21^{\text {Waf1/Cip1 }}$. Our findings suggest that LRRC42 overexpression as well as its interaction with LRRC42-GATAD2B might play essential roles in lung carcinogenesis, and be a promising molecular target for lung cancer therapy.
\end{abstract}

\section{Introduction}

Lung cancer is the leading cause of cancer-related death worldwide. Despite some advances in cancer diagnostics and recent improvements in its treatment, the overall 5-year survival rate is still only $15 \%$ (1). Several oncogenic alterations, such as KRAS and EGFR mutations, and EML4-ALK fusion genes as well as inactivation of tumor suppressor gene

Correspondence to: Professor Yusuke Nakamura, Department of Medicine and Surgery, The University of Chicago, Knapp Center for Biomedical Discovery, 900 E. 57th Street, KCBD6130, Chicago, IL 60637, USA

E-mail: ynakamura@bsd.uchicago.edu

Key words: oncogene, cancer-testis antigen, therapeutic target, lung cancer, novel molecular target of TP53 in lung cancer have been reported, however the precise molecular mechanisms of pulmonary carcinogenesis are still far from fully understood (2). Although several molecular targeted-drugs such as gefitinib, bevacizumab and crizotinib have been approved for lung cancer treatment, the portion of patients who are able to have the benefit of these drugs is still limited and several serious adverse reactions such as interstitial pneumonia by gefitinib and hemorrhage by bevacizumab have been reported $(3,4)$. Hence, the development of molecular targeted agents providing better clinical benefits with less adverse events are eagerly required.

Systematic analysis of expression levels of thousands of genes using a cDNA microarray is an effective technique to identify molecules involved in carcinogenic pathways (5); some of such genes or their gene products may be good molecular targets for the development of novel therapies and/or cancer biomarkers. To isolate potential molecular targets for diagnosis, treatment, and/or prevention of lung carcinomas, we performed a genome-wide analysis of gene expression profiles of tumor tissues from 120 lung cancer cases by means of cDNA microarray consisting of 27,648 genes or expressed sequence tags (ESTs) (6-10). Among the transactivated genes, we identified LRRC42 (Leucine-rich repeat containing 42) as a potential therapeutic target for lung cancer. LRRC42 protein contains two leucine-rich repeats (LRRs) which are widespread structural motifs comprising 20-30 amino acids with a characteristic repetitive sequence pattern rich in leucine residues. Leucine-rich repeat domains are built from tandems of two or more repeats and form curved solenoid structures that are particularly suitable for protein-protein interactions. LRR-containing proteins participate in many important biological processes, including plant and animal immunity, hormone-receptor interactions, cell adhesion, signal transduction, regulation of gene expression and apoptosis (11-14). However, the pathophysiological roles of LRRC42 in cancer cells have not been reported. Herein we report identification of LRRC42 as a potential therapeutic target and also provide evidence that LRRC42 could interact with GATAD2B (GATA zinc finger domain containing 2B) and MBD3 (Methyl-CpG-binding domain protein 3) proteins that are likely to play a significant role in human pulmonary carcinogenesis. 


\section{Materials and methods}

Lung cancer cell lines and tissue samples. The human lung cancer cell lines used in this study were as follows: A549, NCI-H1373, NCI-H1781, SKMES-1, NCI-H520, NCI-H1703, NCI-H2170 and DMS114 were distributed from America Type Culture Collection (ATCC, Manassas, VA, USA). LC319 was kindly provided from Aichi Cancer Center (Aichi, Japan). PC14 was obtained from RIKEN BioRsource Center (Ibaraki, Japan). LU61 and LX1 were obtained from Central Institute for Experimental Animals (Kanagawa, Japan). DMS273 was obtained from European Collection of Animal Cell Cultures (ECACC, Salisbury, UK). SBC-3 and SBC-5 were obtained from Japanese Collection of Research Bioresources (JCRB, Osaka, Japan). All cells were grown in monolayers in appropriate medium supplemented with $10 \%$ FCS and were maintained at $37^{\circ} \mathrm{C}$ in atmospheres of humidified air with $5 \%$ $\mathrm{CO}_{2}$. Human small airway epithelial cells (SAEC) were grown in optimized medium purchased from Cambrex Bio Science, Inc. (Walkersville, MD, USA). Primary lung cancer tissue samples were obtained with informed consent as previously described $(6,10)$. This study was approved by individual institutional ethical committees.

Quantitative real-time PCR. Total RNA was extracted from cultured cells using the QIAshredder (Qiagen, Valencia, CA, USA) and RNeasy ${ }^{\circledR}$ Plus mini kit (Qiagen) according to the manufacturer's protocol. Extracted RNAs were reverse transcribed using oligo (dT) primer (Life Technologies, Carlsbad, CA, USA) and SuperScript III (Life Technologies). Quantitative real-time PCR was conducted with the SYBR Green I Master kit on a LightCycler 480 (Roche Diagnostics, Mannheim, Germany) according to the manufacturer's recommendations. Each experiment was done in duplicate. GAPDH was used for normalization of expression levels. For quantitative RT-PCR reactions, specific primers for all human $L R R C 42, G A T A D 2 B$, $M B D 3, p 21^{\text {Wafl/Cipl }}$ and GAPDH were designed as follows: LRRC42, 5'-TTGATCATAGTAACTGCAAGACAGAG-3' and 5'-ACGCTCCCACTGCAGAAC-3'; GARAD2B, 5'-CACCAACCGGCTGAAAAAT-3' and 5'-GCTGCT GTAATCGCTGTTCA-3', MBD3, 5'-ACCATGGACC TCCCCAAG-3' and 5'-CGACAGCAGCGTCTCATC-3'; $p 21^{\text {Wafl/Cipl },} 5^{\prime}$-GACCTGTCACTGTCTTGTACCC-3' and 5'-AAGATCAGCCGGCGTTTG-3'; GAPDH, 5'-ACCATG GGGAAGGTGAAG-3' and 5'-AATGAAGGGGTCATT GATGG-3'.

Immunofluorescence analysis. Cells were plated onto glass coverslips (Becton Dickinson, Mountain View, CA, USA), fixed with $4 \%$ paraformaldehyde, and permeablilized with $0.1 \%$ Triton X-100 in PBS for 5 min at room temperature. Non-specific binding was blocked by $5 \%$ skim milk for $10 \mathrm{~min}$ at room temperature. Cells were then incubated overnight at $4^{\circ} \mathrm{C}$ with primary antibodies for mouse monoclonal anti-Flag antibody (Catalog no. F3165, Sigma, St. Louis, MO, USA) and anti-GATAD2B antibody (Catalog no. HPA017015, ATLAS, Stockholm, Sweden) diluted in PBS containing $1 \%$ BSA. After being washed with PBS, the cells were stained with Alexa Fluor 488-conjugated secondary antibody (Molecular Probes, Eugene, OR, USA) (Fig. 1C), or with Alexa Fluor 488-conju- gated secondary antibody and Alexa Fluor 594-conjugated secondary antibody (Molecular Probes) (Fig. 3C) for $60 \mathrm{~min}$ at room temperature. After another wash with PBS, each specimen was mounted with Vectashield (Vector Laboratories, Burlingame, CA, USA) containing 4', 6'-diamidine-2'-phenylindolendihydrochrolide (DAPI) and visualized with Spectral Confocal Scanning Systems (TSC SP2 AOBS: Leica Microsystems, Wetzlar, Germany).

Northern blot analysis. Human multiple-tissue blots (Clontech, Carlsbad, CA, USA) were hybridized with ${ }^{32} \mathrm{P}$-labeled PCR products of $L R R C 42$. The cDNA probe of $L R R C 42$ was prepared by RT-PCR using following primers: $L R R C 42$, 5'-GACCAGATCGTTCTGCAGTG-3' and 5'-CCTCCCA CACCACAAAAGTA-3'. Prehybridization, hybridization, and washing were performed according to the supplier's recommendations. The blots were autoradiographed at $-80^{\circ} \mathrm{C}$ for 14 days.

RNA interference assay. To evaluate the biological functions of LRRC42 in lung cancer cells, we used small interfering RNA (siRNA) duplexes against the target genes (Sigma). The target sequences of the synthetic oligonucleotides for RNA interference were as follows: control-1: [EGFP, enhanced green fluorescence protein (GFP) gene, a mutant of Aequorea gictoria GFP], 5'-GAAGCAGCACGACUUCUUC-3'; control-2 (LUC, luciferase gene from Photinus pyralis), 5'-CGUACG CGGAAUACUUCGA-3'; si-LRRC42-\#1, 5'-CUUACUA CCUCAGCUCAGA-3'; si-LRRC42-\#2, 5'-GACUUGUUA AAUUCCUAUU-3'; si-GATAD2B, 5'-GCCAAUAGCG AGUUCAUCU-3'; si-MBD3, 5'-CACAGUCGAGGCACG UCAU-3'. Lung cancer cell lines, LC319 and SBC-3, were plated onto $10-\mathrm{cm}$ dishes $\left(5.0 \times 10^{5}\right.$ per dish), and transfected with either of the siRNA oligonucleotides $(50 \mu \mathrm{M})$ using $30 \mu \mathrm{l}$ of Lipofectamine ${ }^{\circledR}$ RNAiMAX (Life Technologies) according to the manufacturers' instructions. After seven days of incubation, the cells were stained by Giemsa solution to assess colony formation, and cell numbers were assessed by Cell Counting Kit-8 (Dojindo Co., Kumamoto, Japan); briefly, Cell Counting Kit-8 solution was added to each dish at concentration of $1 / 10$ volume, and the plates were incubated at $37^{\circ} \mathrm{C}$ for additional $1 \mathrm{~h}$. Absorbance was then measured at $490 \mathrm{~nm}$, and at $630 \mathrm{~nm}$ as a reference, with a 2030 ARVO $^{\text {TM }}$ X3 (PerkinElmer, Courtaboeuf, France).

Anti-LRRC42 antibody. Plasmids expressing partial LRRC42 that contained His-tagged epitopes at their $\mathrm{NH}_{2}$ termini were prepared using pET28 vector (Novagen, Darmstadt, Germany) and primers LRRC42-F (5'-GGAATTCTGGGCTGA CCAGATCGTTCTGC-3') and LRRC42-R (5'-ATAGTTT AGCGGCCGCTTAGTTATTCTGTTCTGTCTCTGACT-3'. Recombinant proteins were expressed in Escherichia coli BL21 codon-plus strain (Stratagene, San Diego, CA, USA) and purified using Ni-NTA (Qiagen) according to the supplier's protocol. The protein was inoculated into rabbits; immune sera were purified on affinity columns according to standard methods. Affinity-purified anti-LRRC42 antibodies were used for western blotting. We confirmed that the antibody was specific to LRRC42 on western blots using lysates from cell lines that had been transfected with LRRC42 expression vector. 

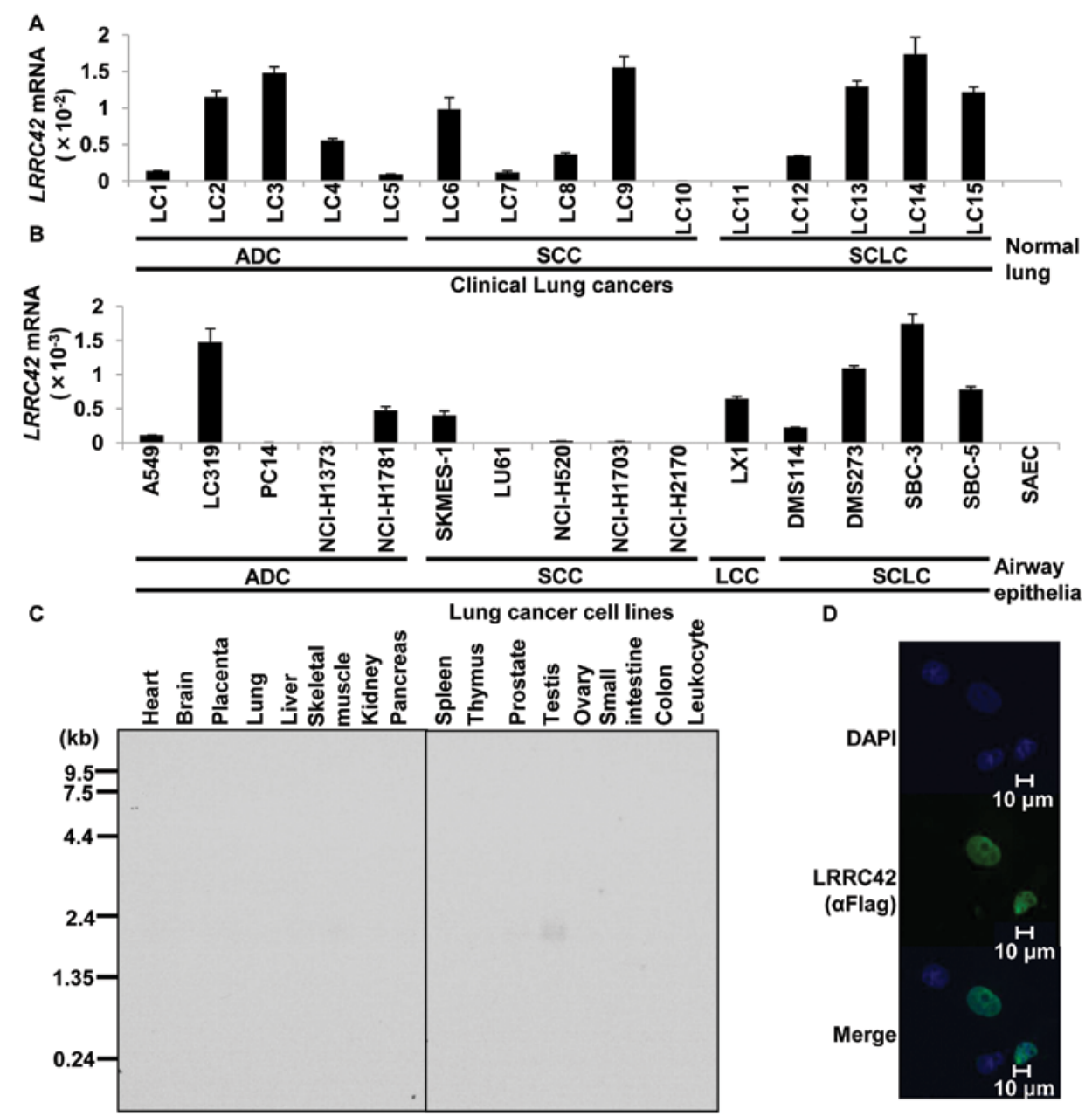

Figure 1. LRRC42 expression in lung cancers and normal tissues. (A) Expression of LRRC42 in clinical NSCLC (non-small cell lung cancer) and SCLC (small cell clung cancer) samples, and normal lung tissues, analyzed by quantitative real-time PCR. mRNA expression levels were normalized by GAPDH expression. The columns and bars represent the mean and SE, respectively. (B) mRNA expression analysis of LRRC42 in lung cancer cell lines by quantitative real-time PCR. The columns and bars represent the mean and SE, respectively. (C) Subcellular localization of exogenous LRRC42 protein in COS-7 cells detected by anti-Flag (green), which were co-stained with DAPI (blue). (D) mRNA expression analysis of LRRC42 in normal human tissues by northern blot analysis.

Western blotting. Cells were lysed with immunoprecipitation assay buffer $[50 \mathrm{mmol} / 1$ Tris-HCl (pH 8.0), $150 \mathrm{mmol} / \mathrm{l} \mathrm{NaCl}$, $1 \%$ NP40, 0.5\% deoxychorate-Na, 0.1\% SDS] containing Phosphatase Inhibitor Cocktail Set II and Protease Inhibitor Cocktail Set III EDTA-Free (Calbiochem, San Diego, CA, USA). Protein samples were separated by SDS-polyacrylamide gels and electroblotted onto Hybond-P PVDF membranes (GE Healthcare Bio-Sciences, Piscataway, NJ, USA). Blots were incubated with a mouse monoclonal anti-Flag antibody, a rabbit anti-Flag antibody (Catalog no. F7425, Sigma), an anti-GATAD2B antibody, an anti-MBD3 antibody (Catalog no. sc-9402, Santa Cruz, Santa Cruz, CA, USA), an antip21 ${ }^{\text {Wafl/Cipl }}$ antibody (Catalog no. 2947S, Cell Signaling, Danvers, MA, USA) and an anti-p53 antibody (Catalog no. sc-126, Santa Cruz, Santa Cruz, CA, USA). Antigen-antibody complexes were detected using secondary antibodies conjugated to horseradish peroxidase (GE Healthcare Bio-Sciences). Protein bands were visualized by enhanced chemiluminescence western blot detection reagents (GE Healthcare Bio-Sciences). LRRC42.T-REx HEK 293 cells that could induce the expression of exogenous LRRC42 (LRRC42-HEK293) cells were generated by Flp-In expression system, where LRRC42 expression is under control of the tetracycline-regulated cytomegalovirus/tet $\mathrm{O}_{2}$ hybrid promoter, and the commercially available Flp-In T-REx 293 host cell line, according to the manufacturer's instructions (Invitrogen, Carlsbad, CA, USA).

Cell growth assay. LRRC42-HEK293 cells were grown for four days in DMEM containing 10\% FBS, Blasticidin S HCl (Life Techonologies; $15 \mu \mathrm{g} / \mathrm{ml}$ ) and Hygromycin B (Invitrogen; $100 \mu \mathrm{g} / \mathrm{ml})$ supplemented with or without doxycycline (Sigma; $100 \mathrm{ng} / \mathrm{ml}$ ). Viability of cells was evaluated by Cell Counting Kit-8.

Coimmunnoprecipitation and matrix-assisted laser desorption/ionizing-time of flight mass spectrometry mapping of LRRC42-associated proteins. Cell extracts from lung cancer cell line SBC-3 which was transfected with LRRC42 expression (carboxyl-terminal Flag-tagged pCAGGS plasmid vector) or mock vector were precleared by incubation at $4^{\circ} \mathrm{C}$ for $1 \mathrm{~h}$ with $80 \mu 1$ of protein $\mathrm{G}$-agarose beads in a final volume of $200 \mu 1$ of immunoprecipitation buffer $(0.5 \% \mathrm{NP} 40,50 \mathrm{mmol} / \mathrm{l}$ 

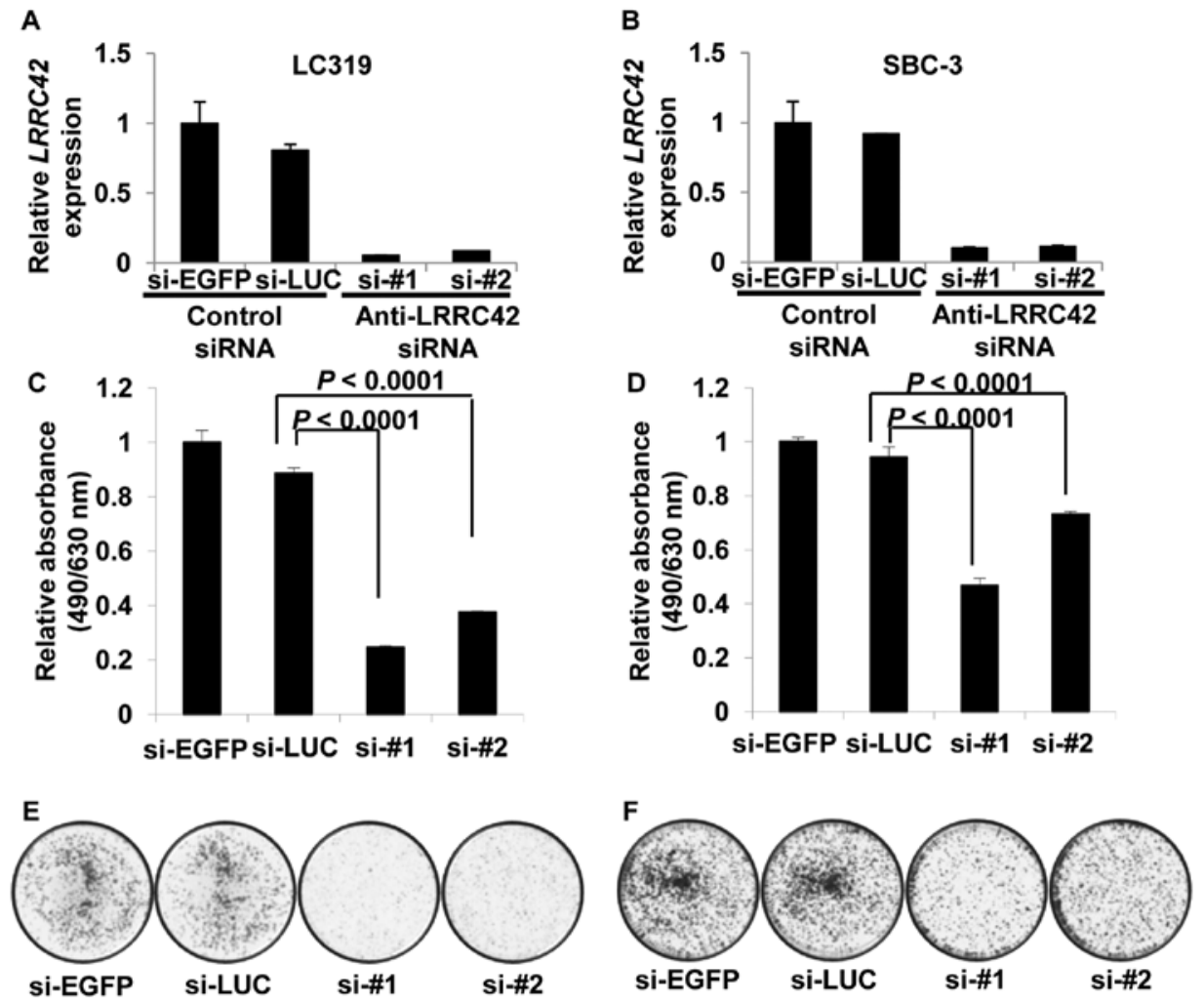

G

H
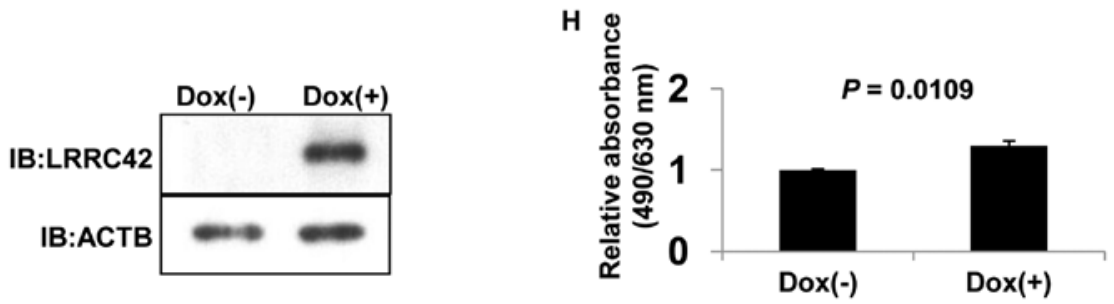

Figure 2. Involvement of LRRC42 in growth and/or survival of lung cancer cells. (A and B) Expression levels of LRRC42 in response to treatment with si-LRRC42 (si-\#1 or si-\#2) or control siRNAs (EGFP or LUC) in LC319 and SBC-3 cells, analyzed by quantitative real-time PCR. The columns and bars represent the mean and SE, respectively. (C and D) Viability of LC319 and SBC-3 cells evaluated by MTT assay in response to treatment with si-LRRC42 (si-\#1 or si-\#2), si-EGFP, or si-LUC. All assays were performed in triplicate using triplicate wells. The columns and bars represent the mean and SE, respectively. (E and F) Colony formation assays of LC319 and SBC-3 cells transfected with specific siRNAs or control siRNAs. (G) Induction of LRRC42 expression in T-REx ${ }^{\text {TM }} 293$ cells with Dox (Doxycycline) by western blot analysis. (H) Assays demonstrating the growth promoting effect of induced LRRC42 expression in T-REx 293 cells. Assays were performed in triplicate using triplicate wells. The columns and bars represent the mean and SE, respectively.

Tris- $\mathrm{HCl}, 150 \mathrm{mmol} / \mathrm{l} \mathrm{NaCl}$ ) in the presence of phosphatase and proteinase inhibitors. After centrifugation at $1,000 \mathrm{rpm}$ for 5 min at $4^{\circ} \mathrm{C}$, the supernatants were incubated at $4^{\circ} \mathrm{C}$ with anti-Flag M2 agarose for $3 \mathrm{~h}$. The beads were then collected by centrifugation at $1,000 \mathrm{rpm}$ for $1 \mathrm{~min}$ and washed six times with $1 \mathrm{ml}$ of each immunoprecipitation buffer. The washed beads were resuspended in $30 \mu 1$ of Laemmli sample buffer and boiled for $5 \mathrm{~min}$, and the proteins were separated using 5\% to 20\% SDS PAGE gels (Bio-Rad Laboratories, Marnes-la-Coquette, France). After electrophoresis, the gels were stained with SilverQuest (Invitrogen). Protein bands specifically found in extracts which was transfected with LRRC42 vector were excised and served for matrix-assisted laser desorption/ionization-time of flight mass spectrometry (MALDI-TOF-MS) analysis (AXIMA-CFR, ShimadzuBiotech, Kyoto, Japan).

Flow cytometry. After transfection of each siRNAs, cells were treated with Aphidicolin (Sigma) at $1 \mu \mathrm{g} / \mathrm{ml}$ for $24 \mathrm{~h}$. Then, the cells were washed with PBS four times and growth medium was added into the dish. The cells were collected in PBS every $3 \mathrm{~h}$ and fixed in $70 \%$ cold ethanol for $30 \mathrm{~min}$. After treatment with $100 \mu \mathrm{g} / \mathrm{ml}$ of RNase (Sigma), the cells were stained with $50 \mu \mathrm{g} / \mathrm{ml}$ propidium iodide (Sigma) in PBS. Flow cytometry was analyzed by using FACScan (Becknman Coulter, Brea, CA, USA). The cells selected from at least 20,000 ungated cells were analyzed for DNA content.

\section{Results}

LRRC42 expression in lung cancers and normal tissues. To identify novel target molecules for the development of therapeutic agents and/or diagnostic biomarkers for lung cancer, we previously performed gene expression profile analysis of 120 lung carcinoma samples using cDNA microarray containing 27,648 genes or expressed sequence tags (6-10), and identified that LRRC42 was significantly transactivated (more than 3 times higher than in their corresponding normal tissues) in 


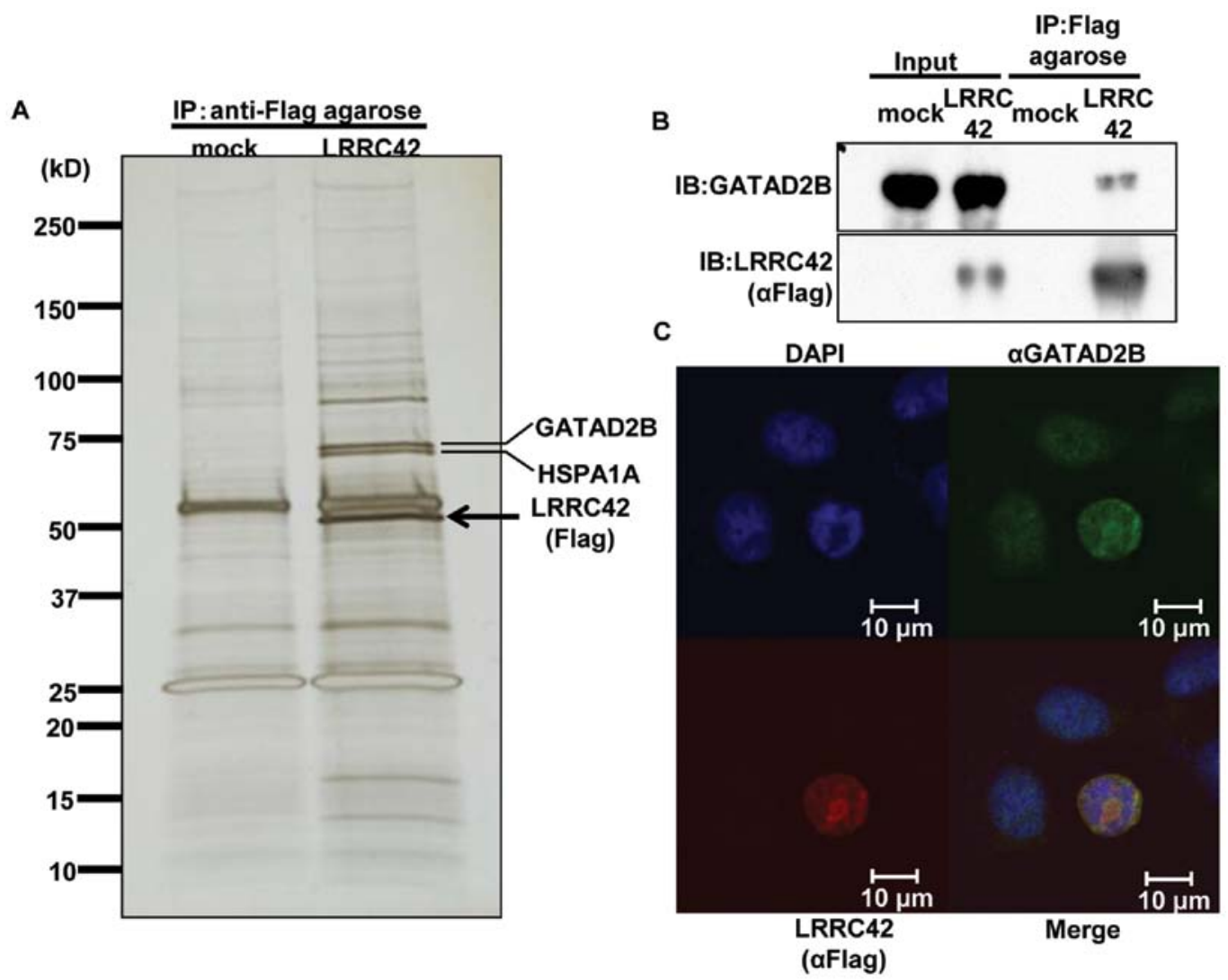

Figure 3. Interaction of LRRC42 with GATAD2B. (A) Silver staining of SDS-PAGE gels that contained immunoprecipitated lysates of lung cancer SBC-3 cells, which were transfected with Flag-tagged LRRC42 expression vector or mock vector, using anti-Flag M2 agarose. (B) Interaction between exogenous LRRC42 and endogenous GATAD2B in SBC-3 cells transfected with LRRC42 expression vector by immunoprecipitation and western blot analysis. (C) Colocalization of LRRC42 and GATAD2B in the nucleus of SBC-3 cells transfected with LRRC42 expression vector, detected by immunocytochemical staining.

$>50 \%$ of 120 lung cancer samples examined. We subsequently confirmed its transactivation by quantitative real-time PCR experiments in lung cancer tissues as well as lung cancer cell lines (Fig. 1A and B). Northern blot analysis with the LRRC42specific probe identified a $1.7-\mathrm{kb}$ transcript only in testis among 16 normal human tissues examined (Fig. 1C). To determine the subcellular localization of LRRC42 protein, we constructed a plasmid expressing LRRC42 (carboxyl-terminal Flag-tagged pCAGGS plasmid vector), transfected it into COS-7 cells and detected exogenous LRRC42 protein in the nucleus of the cells using an anti-flag antibody (Fig. 1D).

Effect of LRRC42 on cell growth. To assess whether LRRC42 is essential for growth or survival of lung cancer cells, we transfected synthetic oligonucleotide siRNAs against LRRC42 into lung adenocarcinoma LC319 and small cell lung cancer SBC-3 cells in which $L R R C 42$ was highly expressed. The mRNA levels of $L R R C 42$ in the cells transfected with si-LRRC42-\#1 or -\#2 were significantly decreased in comparison with those transfected with either of the control siRNAs (Fig. 2A and B). MTT and colony formation assays revealed a significant reduction of cell viability as well as the number of colonies in si-LRRC42-transfected cells (Fig. 2C-F).

To further clarify a potential role of LRRC42 in carcinogenesis, we established HEK293 cells using the Flp-In T-Rex expression system, where LRRC42 expression was under the control of the tetracycline-regulated cytomegalovirus/tet $\mathrm{O}_{2}$ hybrid promoter. MTT assay demonstrated that the growth of the cells treated with doxycycline was enhanced compared with that without doxycycline, indicating the growth promoting activity of LRRC42 protein (Fig. 2G and H).

Interaction and colocalization of LRRC42 with GATAD2B. To elucidate the molecular mechanism of LRRC42 in lung carcinogenesis, we screened a protein(s) that could interact with LRRC42. Lysates of SBC-3 cells which was transfected with LRRC42 expression vector (carboxyl-terminal Flag-tagged pCAGGS plasmid vector) or mock vector were extracted, and immunoprecipitated with anti-Flag M2 agarose. The protein complex was separated by SDS-PAGE and visualized by silver staining (Fig. 3A). A 65-kDa band, which was detectable in lysates of cells transfected with LRRC42 vector, but not in those with mock vector, was extracted. The peptide sequence analysis determined by mass spectrometry indicated the protein to be GATAD2B (GATA zinc finger domain containing $2 \mathrm{~B}$ ) that is known to be a component of the MeCP1 complex that represses transcription through preferential binding, remodeling and deacetylation of methylated nucleosomes (15). We subsequently confirmed interaction between exogenous LRRC42 and endogenous GATAD2B in SBC-3 cells using anti-GATAD2B antibody by co-immunoprecipitation experiment (Fig. 3B). We also conducted immunofluorescence analysis and found colocalization of exogenous LRRC42 with endogenous GATAD2B in the nucleus of SBC-3 cells which were transfected with the LRRC42 expression vector (Fig. 3C). 

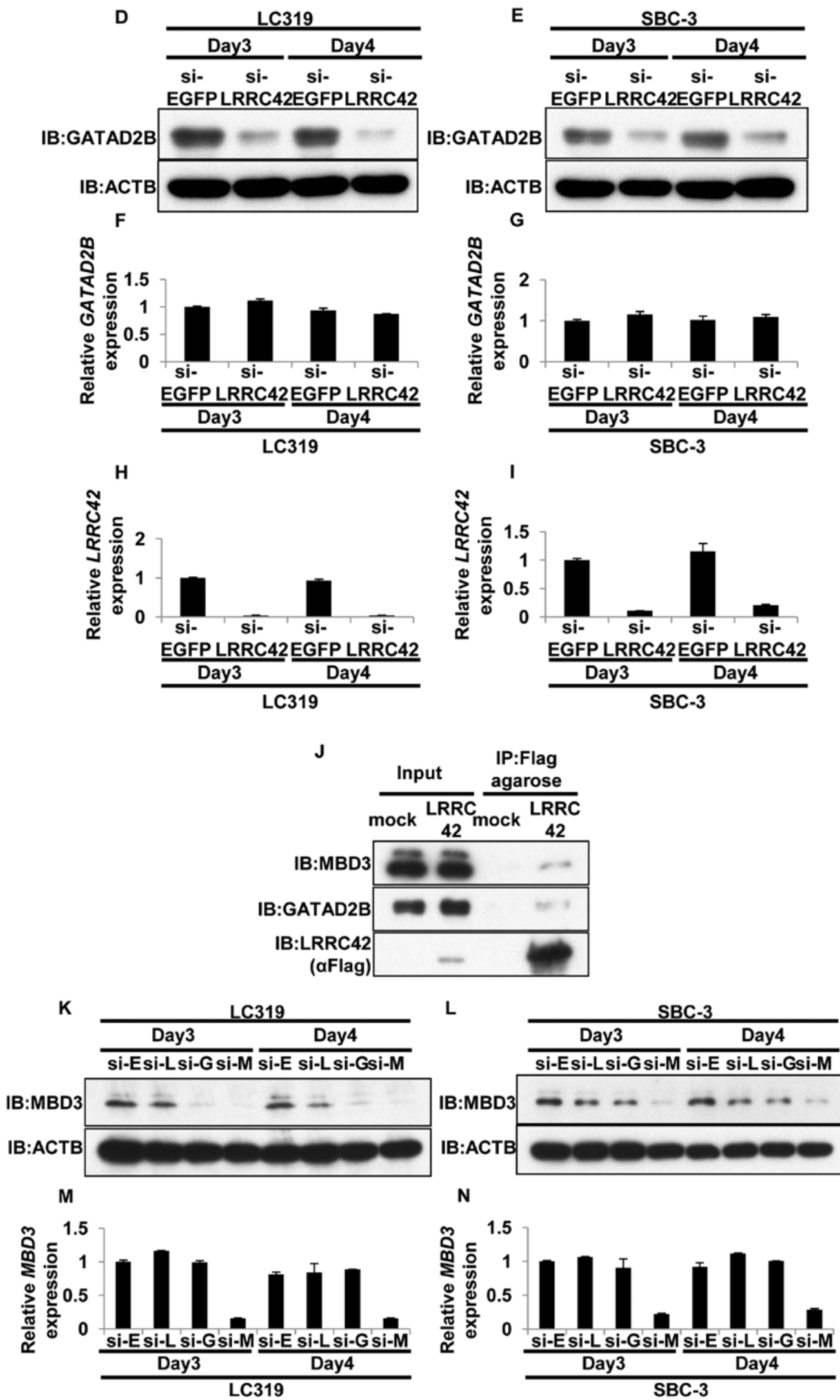

Figure 3 Continued. Interaction of LRRC42 with GATAD2B. (D and E) The level of GATAD2B proteins detected by western blot analysis in LC319 and SBC-3 cells transfected with si-EGFP or si-LRRC42. The columns and bars represent the mean and SE, respectively. (F and G) The level of GATAD2B mRNAs detected by quantitative real-time PCR analysis in LC319 and SBC-3 cells transfected with si-EGFP or si-LRRC42. The columns and bars represent the mean and SE, respectively. (H and I) The level of LRRC42 mRNAs detected by quantitative real-time PCR analysis in LC319 and SBC-3 cells transfected with si-EGFP or si-LRRC42. The columns and bars represent the mean and SE, respectively. (J) Interaction between exogenous LRRC42 and endogenous GATAD2B or MBD3 in SBC-3 cells transfected with LRRC42 expression vector by immunoprecipitation and western blot analysis. (K and L) The level of MBD3 proteins detected by western blot analysis in LC319 and SBC-3 cells transfected with si-EGFP, si-LRRC42, GATAD2B or MBD3. (M and N) The level of MBD3 mRNAs detected by quantitative real-time PCR analysis in LC319 and SBC-3 cells transfected with si-EGFP, si-LRRC42, si-GATAD2B or si-MBD3. The columns and bars represent the mean and SE, respectively. 
To further investigate the biological significance of the interaction between LRRC42 and GATAD2B in cancer cells, we examined the protein level of GATAD2B after suppressing LRRC42 expression in LC319 and SBC-3 cells. Treatment of siRNA oligonucleotides against LRRC42 (si-LRRC42) effectively knocked down the expression of endogenous LRRC42, compared to the control siRNA (si-EGFP). Interestingly, the protein level of GATAD2B was also significantly decreased in cells transfected with si-LRRC42, while the transcript level of GATAD2B was unchanged (Fig. 3D-I). A previous study indicated GATAD2B as a key component of the MeCP1 complex that interacted with MBD3 (15). Furthermore, GATAD2B was shown to possess an ability to target MBD3 protein to specific nuclear loci $(15,46)$. MBD3 was indicated to be recruited to a promoter region of the $21^{\text {Wafl/Cipl }}$ tumor suppressor gene and silence its expression (47). Hence, we have hypothesized that LRRC42 might have a very significant effect on the function of the MeCP1 complex through the interaction with GATAD2B as well as MBD3 (Fig. 3J). We found that knockdown of LRRC42 or GATAD2B reduced the amount of MBD3 protein while no change was observed in mRNA level of MBD3 (Fig. 3K-N).

LRRC42-GATAD2B-MBD3 axis regulates $p 21^{\text {Wafl/Cipl }}$ expression. We then examined the downstream target of the LRRC42-GATAD2B-MBD3 complex in lung cancer cells. As described above, MBD3 might be recruited at the p21 ${ }^{\text {Wafl/Cip1 }}$ promoter and silence its expression (47). Therefore, we firstly assessed the knockdown effect of either of LRRC42, GATAD2B or MBD3 on $\mathrm{p} 21^{\text {Waf1/Cipl }}$ expression by quantitative real-time PCR and western blot analysis. Suppression of either of LRRC42, GATAD2B or MBD3 by siRNA appeared to increase $\mathrm{p} 21^{\text {Wafl/Cip1 } 1}$ at transcriptional and protein levels in LC319 (p53 null) and SBC-3 (p53 wild-type) cells (Fig. 4A-D). However, this effect on $\mathrm{p} 21^{\text {wafl/Cipl }}$ was not clear in other lung cancer cell lines examined (NCI-H1781, NCI-H358, NCI-H1299 and DMS273; data not shown).

Since $\mathrm{p} 21^{\text {Waf } 1 / \mathrm{Cip} 1}$ expression was known to cause the $\mathrm{G}_{0} / \mathrm{G}_{1}$ arrest, we performed FACS (Fluorescence Activated Cell Sorter) analysis to evaluate the knockdown effect on the cell cycle in these cell lines. After synchronization of the cancer cells at $G_{1}$ phase by aphidicolin, we removed aphidicolin from the cell culture medium and monitored the cell cycle progression process (Fig. 4E). The cells transfected with si-EGFP progressed rapidly into the $S$ and $\mathrm{G}_{2} / \mathrm{M}$ phases. However, the cells treated with siRNA against $L R R C 42$, $G A T A D 2 B$ or $M B D 3$ revealed significant delay in entering into $S$ phase although the delay from the $G_{1}$ to $S$ transition in the cells treated with si-MBD3 was less significant to those treated with siLRRC42 or si-GATAD2B. These data implied that the LRRC42-GATAD2B interaction may regulate MBD3 protein as well as the MeCP1 complex, and enhance the growth of cancer cells.

\section{Discussion}

Recent advances in understanding the molecular mechanisms underlying cancer development/progression have driven the design of new therapeutic approaches, termed 'molecular targeted therapies', that selectively interfere with molecules or pathways involved in tumor growth and progression.
Inactivation of growth factors and/or their receptors on tumor cells as well as the inhibition of oncogenic tyrosine kinase pathways that play crucial roles in cancer cells constitute the main rationale of new cancer treatments and also lead to the way for the personalized treatment for individual patients. Small-molecule inhibitors and monoclonal antibodies are at present major components of these targeted approaches for various types of human caner (16). Molecular targeted cancer therapies hold the promise of being very selective to cancer cells, but not affecting normal cells. Hence, they are expected to be less harmful to normal cells, reduce severe side effects, and improve the quality of life of cancer patients. Toward identification of molecular targets for drug development, we had performed whole-genome expression profiles of 120 clinical lung cancer samples using cDNA microarray data and subsequent loss-of-function phenotype analyses by means of RNA interference systems (17-38). On the basis of this approach, we found LRRC42 to be highly over-expressed in the majority of clinical lung cancer cases as well as in most of the lung cancer cell lines examined, while its expression was absent in normal tissues except testis.

Furthermore, we demonstrated that knockdown of LRRC42 expression suppressed the growth of lung cancer cells. In addition, induction of LRRC42 expression by a T-REx system resulted in enhancement of cell growth, suggesting that LRRC42 is likely to be an important growth promoting factor in lung cancer cells and could serve as a valuable target for the development of an anticancer agent for lung cancer.

LRR family members were reported to participate in many biologically important processes such as hormone-receptor interactions, enzyme inhibition, cell adhesion and cellular trafficking. A number of studies clarified the involvement of LRR proteins in early mammalian development (39), development of neuron (40), cell polarization (41), regulation of gene expression (42) and apoptosis (43). It was also shown that LRR domains may be critical for the cell morphology as well as the cytoskeleton dynamics $(44,45)$. In these processes, the LRR motifs are probably essential in mediating protein-protein interactions. However, there has been no report describing the involvement of LRRC42 in human carcinogenesis.

Our data indicated that LRRC42 was able to interact with GATAD2B. GATAD2B is an important component of the $\mathrm{MeCP} 1$ complex that represses transcription of genes through preferential binding to, remodeling, and deacetylating methylated nucleosomes. It has the ability to translocate MBD3 protein to specific nuclear foci $(15,46)$. We demonstrated that LRRC42 could activate this transcription-repressive complex through interacting with and stabilizing GATAD2B and MBD3 proteins. We also revealed that the LRRC42GATAD2B-MBD3 interaction is likely to play a significant role in transcriptional regulation of the cyclin-dependent kinase inhibitor $\mathrm{p} 21^{\text {Wafl/Cip1 }}$ which is a well-known tumor suppressor and an inhibitor of cell cycle progression from $\mathrm{G}_{1}$ to $\mathrm{S}$ phase. $\mathrm{p} 21^{\text {Wafl/Cipl }}$ negatively regulates DNA replication through the interaction with PCNA (Proliferative cell nuclear antigen), and also binds to the CDK (Cyclin-dependent kinase) complex and inhibits the $\mathrm{G}_{1}-\mathrm{S}$ transition (48).

Several transcriptional regulators, activators or repressors of $\mathrm{p} 21^{\text {Waf } 1 / \text { Cipl }}$ have been reported. p53 is an activator of $\mathrm{p} 21^{\text {Wafl/Cipl }}$ and the activation of the $\mathrm{p} 53-\mathrm{p} 21^{\text {Waf1/Cip1 }}$ pathway is 
A

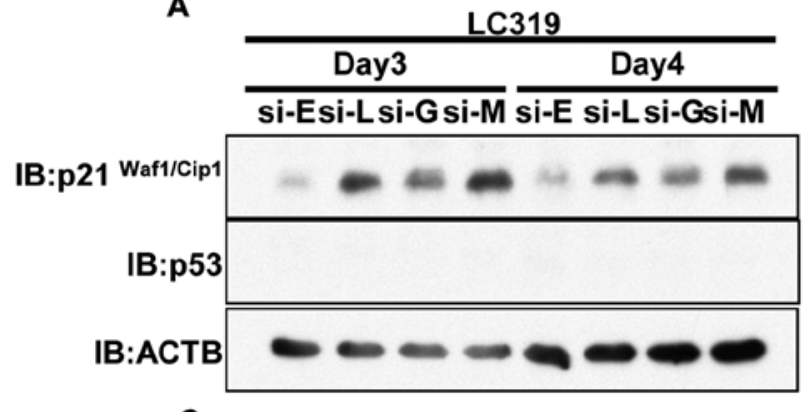

C

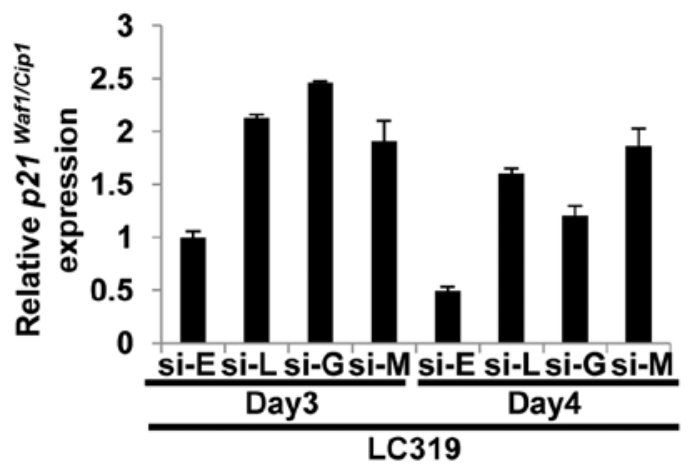

B

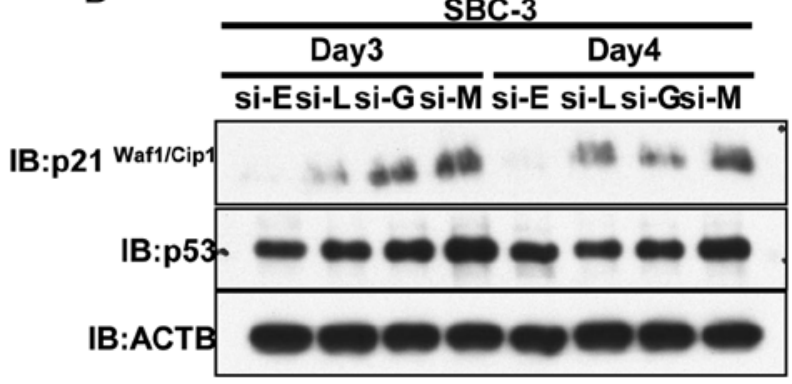

D

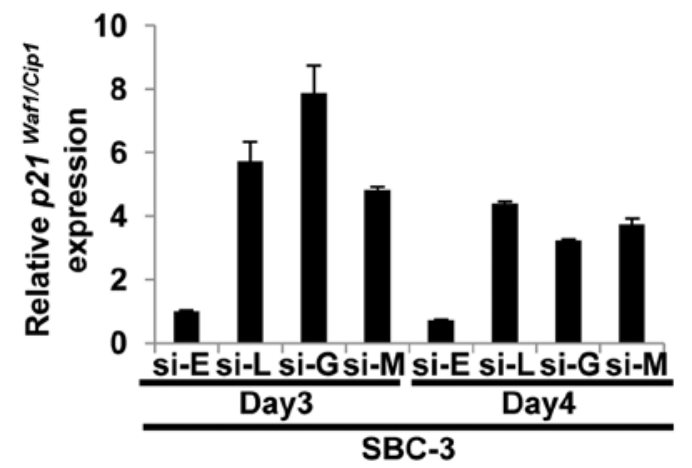

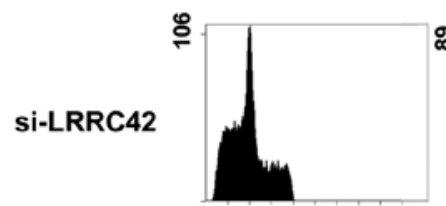
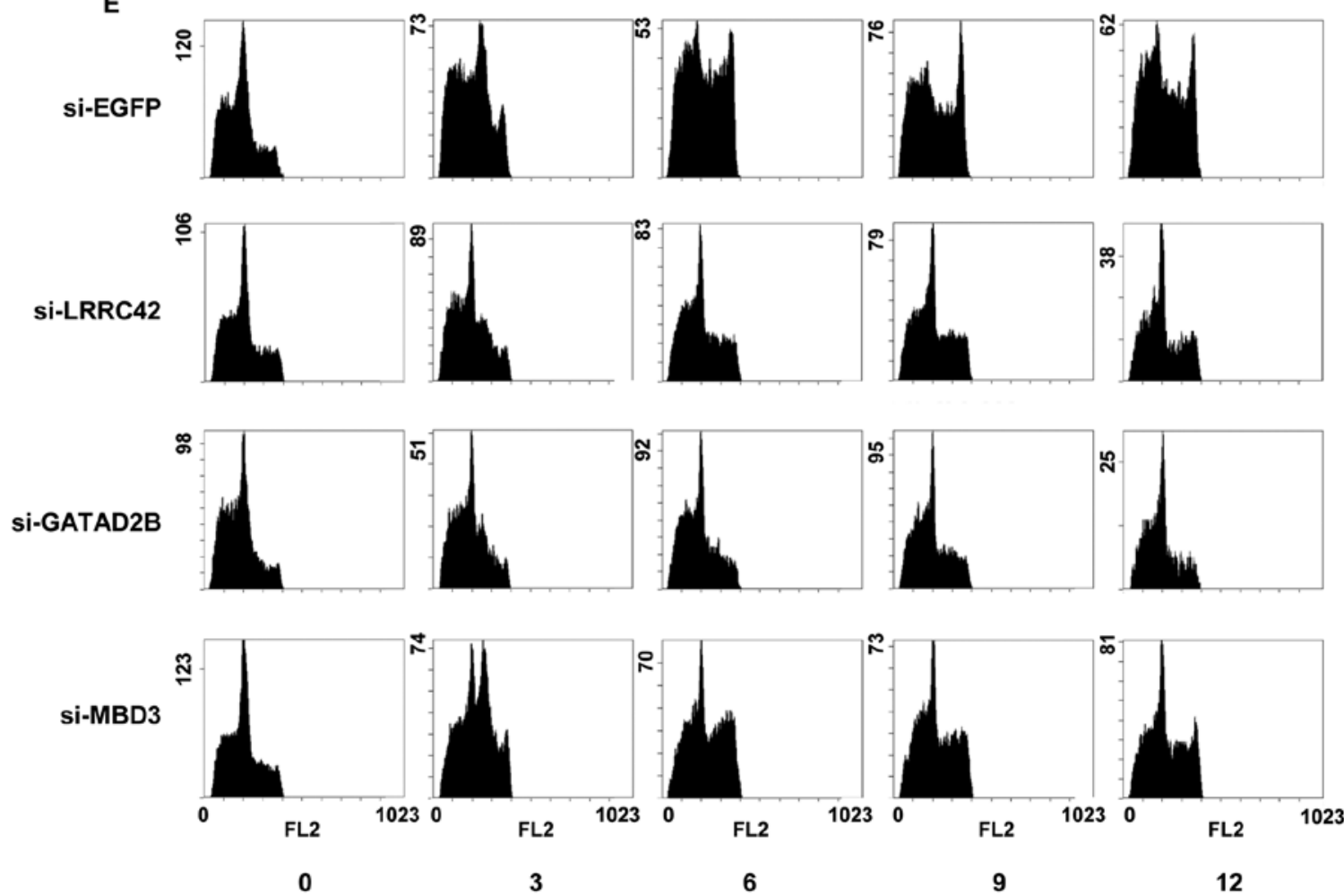

h

Figure 4. Induction of $\mathrm{p} 21^{\text {Wafl/Cipl }}$ and the effect on cell cycle progression by knockdown of LRRC42, GATAD2B or MBD3. (A and B) Expression levels of $\mathrm{p} 21^{\text {Waf } / / \text { Cipl }}$ protein after suppression of LRRC42, GATAD2B or MBD3 by siRNA in LC319 and SBC-3 cells. (C and D) Expression levels of p21 ${ }^{\text {Wafl/Cip1 }}$ mRNA after suppression of LRRC42, GATAD2B or MBD3 by siRNA in LC319 and SBC-3 cells. The columns and bars represent the mean and SE, respectively. (E) Histogram of the cell cycle phases after treatment with si-EGFP, si-LRRC42, si-GATAD2B or MBD3 in SBC-3 cells.

critically important when cells need to arrest the cell cycle and repair the DNA damage. Myc, one of $\mathrm{p} 21^{\text {Wafl/Cip1 }}$ repressors, was reported to induce the expression of AP4 (Transcription Factor AP-4) that has the ability to repress $\mathrm{p} 21^{\text {Wafl/Cipl }}$ expression. Myc may also repress $\mathrm{p} 21^{\text {Waf } 1 / \text { Cipl }}$ expression through the interaction with MIZ1 (ZBTB17) (49). MBD3 is known to be one of the repressors which directly regulate $\mathrm{p} 21^{\text {Wafl/Cip1 }}$ expression and play important roles in oncogenic transformation and proliferation (50). FACS analysis clearly demonstrated that suppression of $M B D 3$ caused the delay of $\mathrm{G}_{1}-\mathrm{S}$ transition 
although its effect was not as significant as the knockdown of LRRC42 or GATAD2B.

In conclusion, human LRRC42 is essential for growth and survival of lung cancer cells. Our data imply that targeting LRRC42 and/or the LRRC42-GATAD2B interaction may be a good approach for development of new treatment of lung cancer with specific activity and minimum toxicity.

\section{Acknowledgements}

This study was supported in part by Grant-in-Aid for Scientific Research (B) and Grant-in-Aid for Scientific Research on Innovative Areas from The Japan Society for the Promotion of Science to Y.D. Y.D. is a member of Shiga Cancer Treatment Project supported by Shiga Prefecture (Japan).

\section{References}

1. Jemal A, Siegel R, Xu J and Ward E: Cancer statistics, 2010. CA Cancer J Clin 60: 277-300, 2010.

2. Daigo Y, Takano A, Teramoto K, Chung S and Nakamura Y: A systematic approach to the development of novel therapeutics for lung cancer using genomic analyses. Clin Pharmacol Ther 94: 218-223, 2013.

3. Inoue A, Saijo Y, Maemondo M, et al: Severe acute interstitial pneumonia and gefitinib. Lancet 361: 137-139, 2003.

4. Sandomenico C, Costanzo R, Carillio G, et al: Bevacizumab in non small cell lung cancer: development, current status and issues. Curr Med Chem 19: 961-971, 2012.

5. Daigo Y and Nakamura Y: From cancer genomics to thoracic oncology: discovery of new biomarkers and therapeutic targets for lung and esophageal carcinoma. Gen Thorac Cardiovasc Surg 56: 43-53, 2008.

6. Kikuchi T, Daigo Y, Katagiri T, et al: Expression profiles of non-small cell lung cancers on cDNA microarrays: identification of genes for prediction of lymph-node metastasis and sensitivity to anti-cancer drugs. Oncogene 22: 2192-2205, 2003.

7. Kakiuchi S, Daigo Y, Tsunoda T, Yano S, Sone S and Nakamura Y: Genome-wide analysis of organ-preferential metastasis of human small cell lung cancer in mice. Mol Cancer Res 1: 485-499, 2003.

8. Kakiuchi S, Daigo Y, Ishikawa N, et al: Prediction of sensitivity of advanced non-small cell lung cancers to gefitinib (Iressa, ZD1839). Hum Mol Genet 13: 3029-3043, 2004.

9. Kikuchi T, Daigo Y, Ishikawa N, et al: Expression profiles of metastatic brain tumor from lung adenocarcinomas on cDNA microarray. Int J Oncol 28: 799-805, 2006.

10. Taniwaki M, Daigo Y, Ishikawa N, et al: Gene expression profiles of small-cell lung cancers: molecular signatures of lung cancer. Int J Oncol 29: 567-575, 2006.

11. Bella J, Hindle KL, McEwan PA and Lovell SC: The leucine-rich repeat structure. Cell Mol Life Sci 65: 2307-2333, 2008.

12. Chai L, Dai L, Che Y, et al: LRRC19, a novel member of the leucine-rich repeat protein family, activates NF- $\kappa \mathrm{B}$ and induces expression of proinflammatory cytokines. Biochem Biophys Res Commun 388: 543-548, 2009.

13. Kajava AV: Structural diversity of leucine-rich repeat proteins. J Mol Biol 277: 519-527, 1998.

14. Pancer $Z$ and Cooper MD: The evolution of adaptive immunity. Annu Rev Immunol 24: 497-518, 2006.

15. Feng Q, Cao R, Xia L, Erdjument-Bromage H, Tempst P and Zhang Y: Identification and functional characterization of the p66/p68 components of the MeCP1 complex. Mol Cell Biol 22: 536-546, 2002

16. Ciavarella S, Milano A, Dammacco F and Silverstris F: Targeted therapies in cancer. BioDrugs 24: 77-88, 2010.

17. Suzuki C, Daigo Y, Ishikawa N, et al: ANLN plays a critical role in human lung carcinogenesis through the activation of RHOA and by involvement in the phosphoinositide 3-kinase/AKT pathway. Cancer Res 65: 11314-11325, 2005.

18. Ishikawa N, Daigo Y, Takano A, et al: Characterization of SEZ6L2 cell-surface protein as a novel prognostic marker for lung cancer. Cancer Sci 97: 737-745, 2006.
19. Kato T, Sato N, Takano A, et al: Activation of placenta-specific transcription factor distal-less homeobox 5 predicts clinical outcome in primary lung cancer patients. Clin Cancer Res 14: 2363-2370, 2008

20. Dunleavy EM, Roche D, Tagami H, et al: HJURP is a cell-cycledependent maintenance and deposition factor of CENP-A at centromeres. Cell 137: 485-497, 2009.

21. Hirata D, Yamabuki T, Miki D, et al: Involvement of epithelial cell transforming sequence- 2 oncoantigen in lung and esophageal cancer progression. Clin Cancer Res 15: 256-266, 2009.

22. Sato N, Koinuma J, Fujita M, et al: Activation of WD repeat and high-mobility group box DNA binding protein 1 in pulmonary and esophageal carcinogenesis. Clin Cancer Res 16: 226-239, 2010.

23. Sato N, Koinuma J, Ito $\mathrm{T}$, et al: Activation of an oncogenic TBC1D7 (TBC1 domain family, member 7) protein in pulmonary carcinogenesis. Genes Chromosomes Cancer 49: 353-367, 2010.

24. Nguyen MH, Koinuma J, Ueda K, et al: Phosphorylation and activation of cell division cycle associated 5 by mitogen-activated protein kinase play a crucial role in human lung carcinogenesis. Cancer Res 70: 5337-5347, 2010.

25. Takano A, Ishikawa N, Nishino R, et al: Identification of nectin-4 oncoprotein as a diagnostic and therapeutic target for lung cancer. Cancer Res 69: 6694-6703, 2009.

26. Sato N, Yamabuki T, Takano A, et al: Wnt inhibitor Dickkopf-1 as a target for passive cancer immunotherapy. Cancer Res 70: 5326-5336, 2010.

27. Mizukami Y, Kono K, Daigo Y, et al: Detection of novel cancertestis antigen-specific T-cell responses in TIL, regional lymph nodes, and PBL in patients with esophageal squamous cell carcinoma. Cancer Sci 99: 1448-1454, 2008.

28. Harao M, Hirata S, Irie A, et al: HLA-A2-restricted CTL epitopes of a novel lung cancer-associated cancer testis antigen, cell division cycle associated 1 , can induce tumor-reactive CTL. Int J Cancer 123: 2616-2625, 2008

29. Kono K, Mizukami Y, Daigo Y, et al: Vaccination with multiple peptides derived from novel cancer-testis antigens can induce specific T-cell responses and clinical responses in advanced esophageal cancer. Cancer Sci 100: 1502-1509, 2009.

30. Yokomine K, Senju S, Nakatsura T, et al: The forkhead Box M1 transcription factor as a candidate of target for anti-cancer immunotherapy. Int J Cancer 126: 2153-2163, 2010.

31. Tomita Y,Imai K, Senju S, et al: A novel tumor-associated antigen, cell division cycle 45-like can induce cytotoxic T-lymphocytes reactive to tumor cells. Cancer Sci 102: 697-705, 2011.

32. Aragaki M, Takahashi K, Akiyama $\mathrm{H}$, et al: Characterization of a cleavage stimulation factor, 3' pre-RNA, subunit 2, $64 \mathrm{kDa}$ (CSTF2) as a therapeutic target for lung cancer. Clin Cancer Res 17: 5889-5900, 2011.

33. Nishino R, Takano A, Oshita $\mathrm{H}$, et al: Identification of Epstein-Barr virus-induced gene 3 as a novel serum and tissue biomarker and a therapeutic target for lung cancer. Clin Cancer Res 17: 6272-6286, 2011.

34. Masuda $\mathrm{K}$, Takano A, Oshita $\mathrm{H}$, et al: Chondrolectin is a novel diagnostic biomarker and a therapeutic target for lung cancer. Clin Cancer Res 17: 7712-7722, 2011.

35. Fujitomo T, Daigo Y, Matsuda K, Ueda K and Nakamura Y: Critical function for nuclear envelope protein TMEM209 in human pulmonary carcinogenesis. Cancer Res 72: 4110-4118, 2012.

36. Koinuma J, Akiyama H, Fujita M, et al: Characterization of an Opa interacting protein 5 involved in lung and esophageal carcinogenesis. Cancer Sci 103: 577-586, 2012.

37. Nguyen MH, Ueda K, Nakamura Y and Daigo Y: Identification of a novel oncogene, $M M S 22 L$, involved in lung and esophageal carcinogenesis. Int J Oncol 41: 1285-1296, 2012.

38. Oshita H, Nishino R, Takano A, et al: RASEF is a novel diagnostic biomarker and a therapeutic target for lung cancer. Mol Cancer Res 11: 937-951, 2013.

39. Tong ZB, Nelson LM and Dean J: Mater encodes a maternal protein in mice with a leucine-rich repeat domain homologous to porcine ribonuclease inhibitor. Mamm Genome 11: 281-287, 2000.

40. Mutai H, Toyoshima Y, Sun W, Hattori N, Tanaka S and Shiota K: PAL31, a novel nuclear protein, expressed in the developing brain. Biochem Biophys Res Commun 274: 427-433, 2000.

41. Bilder D and Perrimon N: Localization of apical epithelial determinants by the basolateral PDZ protein Scribble. Nature 403: 676-680, 2000. 
42. Linhoff MW, Harton JA, Cressman DE, Martin BK and Ting JP: Two distinct domains within CIITA mediate self-association: involvement of the GTP-binding and leucine-rich repeat domains. Mol Cell Biol 21: 3001-3011, 2001.

43. Inohara N, Koseki T, del Peso L, et al: Nod1, an Apaf-1-like activator of caspase-9 and nuclear factor-kappaB. J Biol Chem 274: 14560-14567, 1999.

44. Wu H, Maciejewski MW, Marintchev A, Benashski SE, Mullen GP and King SM: Solution structure of a dynein motor domain associated light chain. Nat Struct Biol 7: 575-579, 2000.

45. Xu P, Mitchelhill KI, Kobe B, Kemp BE and Zot HG: The myosin-I-binding protein Acan 125 binds the SH3 domain and belongs to the superfamily of leucine-rich repeat proteins. Proc Natl Acad Sci USA 94: 3685-3690, 1997.

46. Brackertz M, Boeke J, Zhang R and Renkawitz R: Two highly related p66 proteins comprise a new family of potent transcriptional repressors interacting with MBD2 and MBD3. J Biol Chem 277: 40958-40966, 2002.
47. Noh EJ,Lim DS and Lee JS: A novel role for methyl CpG-binding domain protein 3 , a component of the histone deacetylase complex, in regulation of cell cycle progression and cell death. Biochem Biophys Res Commun 378: 332-337, 2009.

48. Abbas T and Dutta A: p21 in cancer: intricate networks and multiple activities. Nat Rev Cancer 9: 400-414, 2009.

49. Wu S, Cetinkaya C, Munoz-Alonso MJ, et al: Myc represses differentiation-induced p21CIP1 expression via Miz-1-dependent interaction with the p21 core promoter. Oncogene 22: 351-360, 2003.

50. Reese KJ, Lin S, Verona RI, Schultz RM and Bartolomei MS Maintenance of paternal methylation and repression of the imprinted H19 gene requires MBD3. PLoS Genet 3: e137, 2007. 